\title{
INNOCENCE ET PUISSANCE HEIDEGGER FACE AU PRINCIPE DE RAISON
}

\author{
Pierre CAYE
}

Résumé : Le principe de raison, le fait de rendre raison de toutes choses en les référant à un principe fondamental inquestionné, commande, pour Heidegger, la mobilisation totale et la computation universelle du monde par la technique. La critique de ce principe semble conduire Heidegger à une pensée du délaissement des choses et à une épistémologie de l'innocence, affranchies de tout effet de pouvoir métaphysique, politique ou sociologique. Mais l'innocence est ici ambiguë. Elle rappelle le jeu héraclitéen de l'enfant-roi et annonce le retour d'une souveraineté mystérieuse. Le pouvoir de la technique est conjuré au profit d'une autre puissance non moins impérieuse et absolue, une « surpuissance » qui pourrait bien à son tour signifier, d'une façon plus décisive encore que le devenir-technique de la pensée, l'accomplissement et la fin de la métaphysique.

MoTS-CLÉS : métaphysique, principe de raison, acte, puissance, système, Aristote, Gentile, Heidegger.

ABSTRACT: The principle of reason, that is accounting for all things by referring them to an unquestioned, fundamental principle, commands for Heidegger the total mobilization and universal computation of the world by the technical. The critique of this principle seems to lead Heidegger toward a thinking of the relinquishment of things and an epistemology of innocence, freed from any effect of metaphysical, political, or sociological power. That innocence is however ambiguous. It recalls the Heraclitean play of the child-king and announces the return of a mysterious sovereignty. The power of the technical is warded off for the benefit of another power, not less commanding and absolute, a "superpower " which in its turn could effectively mean, in a still more decisive way than the becoming-technical of thinking, the accomplishment and the end of metaphysics.

KEYWORDs: metaphysics, principle of reason, act, power, system, Aristotle, Gentile, Heidegger. 
ZuSAMMENFASSUNG : Das Vernunftprinzip, d.h. die Tatsache, daß alles durch ein Grundprinzip, das nicht in Frage gestellt wird, erklärt werden soll, ist nach Heidegger gleichbedeutend mit der totalen Mobilmachung und der universellen Berechnung der Welt durch die Technik. Die Kritik dieses Prinzips scheint Heidegger dazu zu veranlassen, die Dinge aufzugeben und sich einer Epistemologie der Unschuld zu verschreiben, bei der Metaphysik, Politik und Soziologie wirkungslos sind. Aber die Unschuld ist in diesem Fall zweideutig. Sie erinnert an Heraklits Spiel vom Königskind und kündigt die Rückkehr einer mysterieusen Souveränität an. Die Macht der Technik wird überwunden zugunsten einer anderen, nicht weniger absoluten und beherrschenden Macht, einer "Übermacht", die ihrerseits auf noch entscheidendere Weise als die Technisierung der Dinge zur Überwindung und zum Ende der Metaphysik führen kann.

STICHWÖRTER: Metaphysik, Vernunftprinzip, Akt, Kraft, System, Aristoteles, Gentile, Heidegger.

RiASSUNTO: Il principio di ragione, il fatto di rendere ragione a tutte le cose riferendole a un principio fondamentale non interrogato, comanda, secondo Heidegger, la mobilitazione e il calcolo universale del mondo da parte della tecnica. La critica di questo principio sembra condurre Heidegger a un'idea di abbandono delle cose e ad un'epistemologia dell'innocenza, affrancate da ogni effetto di potere metafisico, politico o sociologo. Ma l'innocenza e qui ambigua. Ricorda il gioco eracliteo del bambino-re e annuncia il ritorno di un potere misterioso. Il potere della tecnica è scongiurato a vantaggio di un'altra potenza non meno imperiosa ed assoluta, una "sovrappotenza " che potrebbe a suo modo stare a significare, in una maniera più forte ancora del divenire-tecnico del pensiero, il compimento e la fine della metafisica.

Parole-Chlave: metafisica, principio di ragione, atto, potenza, sistema, Aristotele, Gentile, Heidegger.

Pierre CAYE, ancien élève de l'École normale supérieure, chargé de recherches au CNRS, travaille sur le devenir-praxis des textualités métaphysiques. Il vient de publier Empire et décor. L'architecture vitruvienne et la question de la technique à l'âge humaniste et classique (Paris, Vrin, 1999).

Adresse: Centre d'histoire de la philosophie moderne, UPR 75 CNRS, BP 8, Bât. N, 7 rue Guy-Moquet, F-94801 Villejuif Cedex.

Courrier électronique : viterbi@infobiogen.fr 
La remise en cause de la raison avec ses processus analytiques et apodictiques fait partie des gestes les plus anciens et les plus fondateurs de la philosophic. La distinction que fait Platon entre nous et dianoia, intelligence et raisonnement, ou l'impossibilité que rencontre selon Aristote le raisonnement à justifier ses axiomes au risque de la pétition de principe ont tôt marqué ses limites à la raison démonstrative.

Il n'y a ici nul irrationalisme, romantisme ou mysticisme. Une telle remise en cause n'a pour but au contraire que d'accroître les pouvoirs de la raison, de lui ouvrir de nouvelles possibilités, de la libérer du carcan de ses objets et de ses opérations formels. C'est ce que la raison se doit à ellemême, ce par quoi elle se comprend et prend sa propre mesure.

"Tous ceux des savoirs qui ont du prix réclament un complément de bavardage et de rêverie spéculative concernant la Nature, car c'est bien de là que s'introduisent en eux la sublimité de pensée qui les caractérise et la perfection de leurs ouvres à tous égards ${ }^{1}$. »

À ce titre, la critique que porte Heidegger contre la rationalité contemporaine et la technicisation du monde qu'elle provoque s'inscrit dans une longue tradition de pensée. Comme ses prédécesseurs, Heidegger ne cherche par cette critique qu'à ouvrir les champs du savoir : il n'y a chez lui aucune fascination pour la nescience. Cependant, sa critique prend une autre proportion. Sous la figure du « principe de raison ", la raison n'est plus limitée au seul domaine de la logique censée expliquer les modes de fonctionnement de la pensée; la raison innerve toutes choses et les rend présentes à l'existence : elle déborde ses limites originelles pour devenir un concept métaphysique. La raison ici n'est plus au service de la critique de la métaphysique, mais participe au contraire à l'instauration de ses nouveaux dogmes. Critiquer la raison ne signifie plus alors revenir à la doxa, aux traditions ou aux charmes de la divagation, mais poursuit jusqu'au bout le projet Aufklärer qui ouvre le champ du savoir en démantelant les dispositifs dogmatiques de la métaphysique. Encore faut-il comprendre, d'une part, en quoi la raison, sous la figure du « principe de raison », participe à la réinstauration dogmatique de la métaphysique et, d'autre part, ce que signifie démanteler ou plus exactement « détruire » la métaphysique. C'est tout le sens de l'«épistémologie » heideggérienne qui est ici en jeu.

1. Platon, Phèdre, 20 a. 
Pour répondre à ces deux questions, quatre thèses sur le système peuvent être formulées, puisque le principe de raison - tel du moins qu'il se présente sous sa formule canonique énoncée par Leibniz: nihil est sine ratione $^{2}$ - est étroitement lié dans l'histoire de la métaphysique à la constitution du système. Les deux premières thèses portent sur l'essence du système en général, et les deux dernières sur le rapport que Heidegger entretient avec cette notion.

Le système se définit au premier chef comme l'articulation fondamentale du principe, du monde et de l'homme. Dans cette articulation, l'homme joue un grand rôle parce qu'il en est non pas le fondement, mais la copule ou le nœud (copula mundi, vinculum universi), c'est-à-dire le point de passage par lequel le principe condescend au monde et quitte sa position d'extériorité, tandis qu'à son tour, par cette même voie, le mouvement ou la marche du monde renouvelle le principe.

La tâche essentielle de la métaphysique a consisté, depuis Nicolas de Cues du moins, à élaborer les concepts nécessaires et suffisants pour rendre pensable l'articulation entre le principe, le monde et l'homme et pour thématiser leurs relations fondamentales. L'histoire de la métaphysique se résume en grande partie à la succession, à la fois respectueuse et conflictuelle, des différents modèles architectoniques qui ont voulu rendre raison du fonctionnement de cette articulation.

La deuxième thèse porte sur la signification et la finalité du système : le système n'est pas tant un dispositif de connaissance et de savoir, serait-il absolu, qu'une machine à dispenser la force et le pouvoir de l'Être. On a 1'habitude de considérer la notion de système essentiellement sous son aspect épistémologique de méthode et de savoir. En réalité, le système du savoir se contente de définir le mode d'inscription de la copule dans la triade, mais ne peut rendre raison du système dans son ensemble. Le système du savoir n'est qu'un moment dans la constitution de la triade et de son fonctionnement qui relèvent essentiellement de la dimension «politique » de la dispensation de la puissance de l'Être.

Cette seconde thèse s'explique plus clairement si l'on aborde la question généalogique de l'origine de cette construction métaphysique qu'est le sys-

2. Opuscules et fragments inédits de Leibniz, éd. Louis Couturat, Paris, Félix Alcan, 1903, p. 515. 
tème et la raison de son apparition. Pourquoi le système? À quel problème métaphysique répond-il ? Pourquoi, enfin, la métaphysique a-t-elle ressenti la nécessité, à un moment de son histoire, de substituer à la physis et au cosmos de l'aristotélisme la triade fondamentale du principe, du monde et de l'homme? C'est en essayant de répondre à ce type de questions que s'impose la nécessité de penser la dimension «politique » du système.

Le système naît de l'incapacité qu'éprouve Aristote de rendre raison du mouvement. La critique peut sembler paradoxale puisque Aristote a précisément introduit le couple de l'acte et de la puissance pour rendre raison du mouvement de la substance et expliquer ainsi la compatibilité du Devenir et de l'Être.

Il est vrai cependant que le mouvement apparaît chez Aristote comme une simple cause auxiliaire au service des causes principales que sont la forme et la fin $^{3}$. Le mouvement aristotélicien se contente de faire cercle entre l'espèce et ses réalisations individuelles. Il ne sert qu'à rendre raison de la distance et de l'intervalle temporel qui règne dans la plénitude cosmique de l'Être. Ce que manque en réalité Aristote, selon Giovanni Gentile, c'est le mouvement en tant que tel, c'est-à-dire le moment précis du en-train-de-se-faire, le processus de l'actuation, l'essence même de la transformation, le mouvement du mouvement : kinesis kineseôs.

«La puissance, la matière c'est du pas encore, tandis que la forme et l'acte c'est du déjà fait, du déjà épuisé. De sorte qu'en analysant l'individu on se trouvera inévitablement devant les deux éléments qui le constituent [à savoir la matière ou puissance d'une part, la forme ou l'acte d'autre part] sans pouvoir en saisir le rapport qui est précisément le processus d'actuation de l'individu ${ }^{4}$."

Pour penser l'essence du mouvement en tant que mouvement, la métaphysique va au cours de son histoire, et en particulier au Moyen Âge, procéder à un certain nombre d'opérations de déplacement de la physiscosmos aristotélicienne qui, petit à petit, vont conduire inéluctablement au système. Opérations qu'il est possible de résumer très brièvement en quatre étapes :

a. La première opération consiste à penser le mouvement comme une trangression de l'ordre des essences et non plus comme sa réalisation. Libéré de ses termes de départ et d'arrivée, le mouvement en tant que tel ne

3. « Le mouvement aristotélicien cesse quand son but est atteint; il n'est donc plus là pour partager la perfection de l'acte qu'avait préparée sa propre imperfection [...] La cause motrice, le mouvement n'a aucune valeur propre, il tire toute sa justification de ce qu'il se propose et de ce qu'il obtient ", Victor GoldschmidT, Le Système stoicien et l'idée de temps, Paris, Vrin, 1989 , p. 94.

4. Giovanni Gentile, L'Esprit, acte pur, trad. franç. Aline Lıon, Paris, Alcan, 1925, p. 57. 
mesure plus une distance à combler entre deux points, mais nous fait accéder à l'expérience du transcendantal, au sens scolastique du terme, c'est-àdire de ce qui nous permet de franchir les frontières entre les ordres, les genres, les espèces et les substances : une métabasis eis allo génon. Le mouvement devient une métabase.

b. La scolastique a complété cette première opération de transgression par une seconde opération qui distingue l'ordre structuré, statique et hiérarchique de l'univers et de ses essences, de l'existence pensée comme mouvement métabasique, fondateur de la dynamique variationnelle du monde.

c. La distinction scolastique de l'essence et de l'existence va à son tour entraîner un déplacement radical de la question théologique, que saint Thomas n'avait certainement pas pressenti lorsqu'il opérait cette fameuse distinction. En effet, sous l'influence de cette distinction, les vieilles théologjes de la création qui insistaient sur l'origine des essences, leur constitution, leur production et leur distinction vont s'effacer au profit des théologies de l'expression qui s'efforcent de leur côté à mettre en lumière les modalités de l'existence et de son déploiement sans rupture entre le producteur et le produit, sans différence entre l'intériorité et l'extériorité du processus, et qui conçoivent donc l'existence comme l'expression directe et sans médiation de la dynamique divine.

d. La théologie de l'expression permet alors de penser le mouvement de métabase non plus comme une simple transgression, mais de façon bien plus décisive comme le principe général du « procès mondial dans lequel et à travers lequel le monde lui-même provient à nouveau, reprend naissance dans ce qui lui est à chaque fois source ${ }^{5} \gg$. C'est le procès lui-même, et non l'architectonique de la triade, qui devient le sujet du système. Le système devient mouvement et mobilité. L'essence du mouvement du monde est donc elle-même mouvement, à la fois Être et Devenir : Être en tant que le mouvement donne l'existence, et Devenir en tant que ce qui donne le mouvement d'exister n'est rien d'autre que mouvement.

Le système peut bien justifier le mouvement du mouvement, mais ne nous dit pas encore en quoi il y va de la force de l'Être et de sa dispensation. Même si l'existence d'un lien entre la question du mouvement et celle de la force se laisse deviner, la conséquence de l'une à l'autre ne va pas de soi. C'est en méditant à nouveau la pensée d'Aristote que l'on peut comprendre cette conséquence ainsi que sa signification et ses enjeux métaphysiques.

Aristote emploie le couple de l'acte et de la puissance pour justifier le mouvement dans le cadre de sa synthèse de l'Être et du Devenir. Mais le

5. Martin HeIDEgGer, Schelling, Le traité de 1809 sur l'essence de la liberté humaine, trad. franç. Jean-François Courtnke, Paris, Gallimard, 1977, p. 107. 
caractère diminué et déficitaire du mouvement chez Aristote réduit à son tour la force du couple de l'acte et de la puissance. Sous la logique du cercle clos de l'espèce et de la réalisation individuelle, chacun des deux moments du couple, au lieu de s'enclencher en une dyade perpétuelle et d'intensifier ainsi le flux de la Vie, s'entre-limite dans le respect de la substance; ici, l'acte ne peut jamais transgresser les limites que lui assignent les conditions spécifiques de la puissance, tandis qu'à son tour la force propre que contient la puissance se trouve arrêtée et étroitement corsetée par la perfection entéléchique de l'acte. Le couple aristotélicien de l'acte et de la puissance apparaît subsumé et déterminé par un autre jeu de concepts : nature, substance, forme et fin. Le devenir-Force de l'Être et de son mouvement est avorté.

La détermination métabasique du mouvement par le système va libérer l'acte et la puissance de leur entre-limitation, et absolutiser chacun des moments de ce couple, tantôt sous la forme classique de la toute-puissance conçue comme omnipossibilité, tantôt sous sa forme idéaliste d'acte pur, de fondation sans fondement, d'avènement non plus du possible, mais de l'impossible, ce que Georges Bataille appelle précisément la Souveraineté. Cependant, cette tendance à l'absolutisation du couple fondamental de l'ontologie qui caractérise l'histoire de la métaphysique n'est pas sans entraîner une dissociation de l'acte et de la puissance entre, d'un côté, l'omnipossibilité qui se passe de tout acte et, de l'autre, l'acte pur qui se passe de toute condition. Mais l'histoire du système n'est pas finie. Le système en son histoire est puissamment aiguillonné par la volonté de reconstituer la synthèse du couple aristotélicien, mais sous une forme absolutisée et intensifiée : non plus le couple de la dunamis et de l'energeia, mais plus radicalement celui de la toute-puissance et de l'acte pur. Tel est le programme qu'annonce Schelling : « Le pouvoir-être, c'est-à-dire l'Être comme pouvoir et comme force, doit être ce qui est perpétuellement acte sans cesser d'être puissance, c'est-à-dire source de l'Etre ${ }^{6}$. » La convertibilité de l'Être, du mouvement et de la force est alors parfaitement assurée par la voie du système.

Si l'on définit donc le système comme l'articulation du principe, du monde et de l'homme, et, en tant que tel, comme le dispositif fondamental de dispensation de la force de l'Être, ou mieux encore de l'Être comme dispensation et donation de force, alors Heidegger reste un penseur du système après sa Khere, comme il l'est déjà - et avec quelle grandeur! dans $\hat{E}$ tre et temps. Il est même possible d'affirmer qu'il va jusqu'au bout

6. Cité par Marc Richir, in Friedrich von Schelling, Philosophie de la mythologie, trad. franç. Alain Pernet, Grenoble, Jérôme Millon, 1994, p. 60. 
du déploiement du système et de sa force, considérée au sens schmittien du terme comme Bewegung ${ }^{\gamma}$ ou pur mouvement, voire comme violence absolue et divine, ce que Walter Benjamin appelle Gewalt ${ }^{8}$. C'est précisément pour penser ce mouvemesl pur et radical que Heidegger met en place la dialectique de l'Ereignis et de la Réserve, accomplissant ainsi de façon absolue et définitive la nouvelle synthèse de l'acte et de la puissance que réclamait Schelling. Par cet accomplissement métaphysique de l'Être et de sa dyade directrice, Heidegger apparaît, plus certainement que Nietzsche, comme le penseur de l'achèvement de la métaphysique dans un dispositif de pouvoir absolu.

C'est dans cette perspective que doit se comprendre la critique heideggérienne du principe de raison. En dénonçant le principe de raison, Heidegger ne remet pas en cause le système en tant que tel, mais simplement un mode bien précis de son fonctionnement, réglé non seu'ement par le principe de raison, mais aussi par ses corollaires, le principe de causalité, d'identité, de non-contradiction: mode qui, pour Heidegger, constitue non pas tant la réalisation du système que son blocage. Heidegger de nouveau reste tributaire de Schelling qui distingue, dans son Système des âges du monde, deux systèmes, un système négatif et un système positif :

«Le mot système a été pris en un bon et en un mauvais sens [...] Au mauvais sens du terme, le système est ce que les médecins grecs nomment le blocage

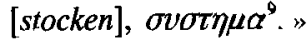

Cette première conception qui renvoie au sustéma des stoìciens marque encore de son sceau, pour Schelling du moins, les systèmes de Fichte et de Hegel. Mais ce n'est pas la seule conception du système possible. Schelling poursuit alors: «Mais système signifie aussi la suite harmonique, le rythme, la tonalité ${ }^{10}$. »Par la critique du principe de raison, Heidegger cherche au premier chef à débloquer le système et à en assouplir le fonctionnement, en retrouvant, par delà la raison et son principe, le sens du rythme, du ton et de l'harmonie qui l'animent. Ce qu'on appelle destruction de la métaphysique se limite en réalité à opérer ce déblocage, pour nous faire accéder au système dans son déploiement libre, absolu et intensifié.

C'est cette quatrième et demière thèse qui va être justifiée ici.

7. Carl Schmirt, Les Trois rypes de pensée juridique, trad. franç. Mira Köller et Dominique SÉglard, Paris, Presses universitaires de France, 1995.

8. Walter BENJAMIN, «Zur Kritik der Gewalt », in Gesammelte Schriften, II-1, hrsg. von Rolf Tiedemann und Hermann SchwePPENHÄUSER, Francfort, Suhrkamp, 1977, p. 179-203.

9. F. von Schelling, System der Weltalter, hrsg. von Siegbent PeEtz, Francfort, Klostermann, 1990 , p. $19-20$.

10. Ibid. 
PUISSANCE

L'analyse du principe de raison par Heidegger se déploie en deux volets. Dans un premier temps, Heidegger instruit un procès en généalogie, une reconnaissance en paternité de ce principe qu'il rattache à la question de la puissance et de sa dispensation «systématique». Puis dans un second temps, par une sorte de renversement dialectique de sa problématique, Heidegger s'attachera à montrer l'épuisement du pouvoir que le principe de raison met ainsi en jeu, et le caractère fictif du système qu'il fait fonctionner.

Même si Le Principe de raison fait partie des derniers grands textes de Heidegger (édité en mars 1957 à partir de ses cours de 1955-1956), il s'inscrit dans un questionnement présent très tôt dans sa méditation, et qui ne cesse de ressurgir. La question du principe de raison nous permet ainsi de suivre le mouvement de sa pensée à travers le temps, sans obligatoirement nous référer à quelque tournant trop dramatique de sa philosophie. En effet, Heidegger y consacre un premier texte dès 1929 : Von Wesen des Grundes que l'on traduit habituellement par Ce qui fait l'Etre-essentiel d'un fondement ou "raison". Dans ce premier texte, Heidegger inscrit le principe de raison dans la problématique du système et de sa triade, au-delà du simple rapport dyadique du sujet et de l'objet qui jusqu'alors servait à expliquer l'apparition et la signification de ce principe dans l'histoire de la métaphysique. Heidegger reste cependant insatisfait de ce premier texte. Cette première étape, à savoir le passage de la dyade noétique à la triade «systématique ", lui semble encore insuffisante pour expliquer la fonction métaphysique du principe. Il considère en effet n'avoir pas encore, dans ce premier texte, pris véritablement la mesure de la «puissance » que le principe de raison mobilise, ni pensé le rapport essentiel que ce principe entretient avec la question métaphysique du pouvoir et de sa dispensation ${ }^{11}$. C'est à cette dernière tâche que se consacre essentiellement le texte de 1957.

Ce texte de 1957 vise deux cibles et deux adversaires. Il constitue d'abord une critique des rationalismes axiomatiques - cercle de Vienne,

11. M. Heidegger, Le Principe de raison, trad. franç. André PrÉAu, Paris, Gallimard, 1962 (cité par la suite comme Le Principe de raison), p. 83 : "Nous autres qui nous nous trouvons ici aujourd'hui, avons-nous déjà senti cette puissance du très puissant principe de raison, l'avons-nous surtout éprouvée comme telle et en outre méditée suffisamment? Si nous ne voulons pas nous leurrer, nous tous nous ne pouvons que répondre non. Nous tous, dis-je, y compris ceux qui, à diverses reprises, se sont déjà formé quelque pensée sur l'Être de la raison. » 
épistémologie néokantienne de l'école de Marbourg, philosophie analytique anglo-saxonne, etc. - et de leur théorie de la vérité prétendument neutre, objective, purement logique, affranchie de tout fantasme métaphysique et de tout effet de pouvoir et de domination. C'est cette fiction angélique, cette prétention à l'innocence - et nous verrons l'importance que prend pour Heidegger la question de l'innocence dans sa réflexion " épistémologique » - que la critique heideggérienne cherche avant tout à dénoncer.

Par le principe de raison, toute chose est appelée à rendre compte et à justifier de son existence. Le pourquoi de la chose, sa raison, son compte se substituent à la chose même. Par cette substitution, le monde devient alors calculable, commutable et mobilisable. Heidegger s'appuie évidemment sur la double signification que la langue latine donne à ratio, à la fois raison et calcul. Mais ce au nom de quoi la chose est justifiée et par cette justification mobilisée dans le calcul du monde reste mystérieux. Pourquoi et au nom de quoi la chose doit ainsi rendre des comptes et se mettre au service de la calculabilité du monde ne va pas de soi. En réalité, le principe de raison est sans raison ${ }^{12}$. Vouloir démontrer le principe de raison consiste à ramener, comme le baron de Münchausen, sa natte sur le devant de la tête et à la tirer pour se sortir de l'eau ${ }^{13}$. En réalité, ce qui justifie ainsi la computation et l'arraisonnement du monde par le calcul du principe de raison est totalement étranger à la raison. La computation arraisonnante du monde relève de la volonté de puissance. Or, la volonté de puissance consiste précisément à vouloir la puissance pour la puissance elle-même, sans projet ni raison. C'est là le coup de force interprétatif de Heidegger: rapprocher les inconciliables, en rattachant le rationalisme axiomatique aux catégories de Nietzsche, qui passe alors pour le penseur de l'irrationnel, et montrer ainsi, derrière leur opposition de façade, la profonde complicité qui unit, par l'intermédiaire de la mobilisation totale du monde et de la volonté de puissance, raison et irraison.

En révélant le lien essentiel qui réunit les deux attitudes extrêmes de la pensée, Heidegger se pose comme la tierce voie du recours. Il démontre combien il est nécessaire, pour fonder à nouveau frais l'épistémologie, de sortir de cette fausse opposition entre, d'une part, la rationalité analytique du logicisme et, d'autre part, l'irrationalité des mythologies, des formations de souveraineté et des conceptions du monde qui inspitent la nouvelle idéo-

12. Le Principe de raison, p. 70 : « Rien n'est sans raison - dit le principe de raison. Rien, pas même le principe de raison, lui certes moins qu'autre chose. À moins toutefois que le principe de raison, ce dont il parle et sa parole elle-même ne soient justement hors du champ d'application du principe de raison. C'est dire en deux mots, que le principe de raison est sans raison. 》

13. Cité in Arthur Schopenhauer, De la quadruple racine du principe de raison (version 1847), trad. franç. François-Xavier Chenet, Paris, Vrin, 1991, p. 156. 
logie allemande des Spengler et autres pseudo-nietzschéens de l'entredeux-guerres. Heidegger réussit ici à pointer l'incapacité où se trouve toute philosophie formaliste, qu'elle s'exerce en morale et en politique aussi bien qu'en épistémologie, d'assumer et de justifier son rapport à la question de la métaphysique et de sa puissance, rapport d'autant plus fort et virulent qu'il est précisément inassumé, voire dénié. Or, nous savons depuis Nietzsche que, pour accomplir la fin de la métaphysique et pour la surmonter, il faut avoir le courage de l'accompagner et de la soutenir jusqu'au bout.

Le rationalisme axiomatique constitue assurément la cible la plus évidente de la critique heideggérienne du principe de raison, mais il n'en est pas pour autant l'adversaire principal. En réalité, Heidegger vise au premier chef la constitution phénoménologique des sciences par Husserl. Husserl est beaucoup moins naif sur cette question que le rationalisme axiomatique et, par bien des points, il annonce le programme de Heidegger. Comme Heidegger, Husserl entreprend de penser l'essence non scientifique de la constitution scientifique, et comme Heidegger il la rattache à la question de la puissance, mais sous une autre forme. Husserl essaie de réinscrire la science objective, abstraite, rationnelle, qui travaille sur le monde mort des êtres de raison, dans son origine humaine et vitale. Il relie la possibilité même de la représentation rationnelle au terreau énergétique de la vie et à l'homme transcendantal considéré comme le résultat de la fusion totale et parfaite de l'ensemble de ses facultés aussi bien sensibles qu'intelligibles. En tant que renvoi au fond vital et humain de l'activité de représentation, la tentative husserlienne conduit à une anthropologie; la philosophie ne devenant elle-même que dans un perpétuel dialogue avec les sciences humaines. C'est évidemment contre cette généalogie anthropologique du savoir et de sa puissance que Heidegger s'élève, comme si Husserl faisait finalement de la volonté de puissance la véritable destination de l'homme, et non pas la simple fatalité d'un dispositif aveugle et inhumain.

C'est pourquoi Heidegger, face à l'anthropologisation de la puissance de la raison, insiste au contraire sur la principialité du principe de raison. Qu'est-ce que le principe ici? Qu'en est-il de sa principialité? Le principe ne se résume pas simplement au fondement. Le fondement n'est qu'un des éléments de la triade systématique. Le principe détermine de façon bien plus générale le mode même de chacun des éléments et de leur articulation réciproque. Le principe rend raison de la configuration du système, de son architectonique. Il est l'horizon de constitution du système qui conditionne l'ensemble de son fonctionnement et de sa signification. Nous allons ainsi examiner très brièvement comment la copule, le monde et le fondement se trouvent tour à tour déterminés de façon bien spécifique par le principe de raison. 
Le principe de raison détermine la copule comme subjectivité. Le sujet n'est pas une substance, le substrat de la pensée, mais apparaît au contraire comme le résultat de l'opération même de l'esprit quand il rend raison. Ainsi, la subjectivité est l'un des modes possibles de l'articulation du système par l'homme : le mode précisément que détermine le principe de raison. Mais qu'est-ce que la subjectivité ? En quoi est-elle le mode rationnel de la copule, en quoi détermine-t-elle la copule telle que le principe de raison l'appelle? La subjectivité, précise Heidegger, n'est pas le subjectif, c'est-à-dire l'affirmation de l'ego, la conscience et l'amour de soi : ici le principe de raison n'a pas encore sa place ${ }^{14}$. La subjectivité, c'est «la conformité à des lois qui est un trait essentiel des raisons livrant la possibilité d'un objet [c'est-à-dire l'objectité] ${ }^{15}$ ». Ce n'est que par la maîtrise des lois de l'objectité que l'homme s'accomplit lui-même, comme ego et plus exactement comme ego transcendantal, face à l'objet qu'il a constitué.

À son tour, ce mode de présence de l'homme aux choses détermine une mondialité spécifique. Par la loi de connaissance ou objectité grâce à laquelle l'homme affirme son ego, la chose se trouve réduite à l'état d'objet. Être objet ne signifie pas seulement se tenir sous la main, à notre disposition. Cette mise à la disposition n'est possible que parce que cet objet n'est rien en soi mais dépend entièrement de son objectité. Les choses n'accèdent à la réalité et ne font monde que par les lois a priori de la connaissance qui conditionnent la possibilité qu'ont ces choses d'être objets d'expérience ${ }^{16}$. La substitution objective de la chose par la raison de sa possibilité de connaissance lui fait perdre son opacité, son impénétrabilité, sa singularité. L'objectité en tant que telle entraîne la communication et la convertibilité des choses entre elles, ce que Heidegger appelle la computation universelle et totale (Universale und totale Verrechnung) qui réduit toute chose à une quantité calculable et l'arraisonne dans ce que Ernst Junger appelle de son côté la mobilisation totale (die totale Mobilmachung) du monde. Sous le règne du principe de raison, la mondialité devient alors dispositif de la technique. L'homme, en faisant le lien entre les lois pures de l'entendement et le monde, assure la substitution de l'objet

14. Le Principe de raison, p. 182 : «La subjectivité n'est rien de subjectif au sens où elle pourrait seulement désigner ce qui n'appartient qu'à un homme individuel, avec toute la contingence de son être particulier et de son goût personnel. »

15. Le Principe de raison, p. 182.

16. Le Principe de raison, p. 176-177: «L'objectivité de l'objet est manifestement ce que l'objet a de plus propre, pourtant elle n'est pas appliquée sur l'objet ou impliquée en lui comme le serait autrement l'une quelconque de ses propriétés. C'est l'objectivité, au contraire, qui s'approprie l'objet, non pas après coup, mais avant même qu'il n'apparaisse comme objet et pour qu'il puisse apparaître comme tel. » 
à la chose et le passage de la physis à la technique. Tel est le mode copulatoire de l'homme qu'implique pour Heidegger la méthode transcendantale.

Cette transformation du monde de la physis en monde de la technique par la subjectivité que l'homme prend en charge n'est possible que parce que la rationalisation et l'objectivation des choses se font sous le mode de la représentation. Représenter une chose c'est la présenter sous le mode de sa condition d'intelligibilité qui, à son tour, se réfère à une condition plus haute, celle de la possibilité de cette intelligibilité, ou plus exactement de la puissance qui détermine notre faculté de comprendre et de représenter. C'est ici que la subjectivité et sa rationalité renvoient à la nécessité du fondement et bouclent la triade du système au-delà de la simple dyade du sujet et de l'objet. Or, comme il a été dit, la puissance que la raison de la subjectivité réclame et à laquelle elle renvoie nécessairement comme à son fondement est sans raison ni projet : elle est volonté de puissance. C'est la volonté de puissance qui explique finalement le devenir-sujet de l'homme, son entreprise d'objectivation des choses et d'organisation du monde en un dispositif technique.

En traçant cette généalogie de la puissance, Heidegger montre, face à Husserl, que la puissance n'est ni consubstantielle ni originaire à l'homme en tant que tel, mais qu'elle relève d'un dispositif complexe et ambigu dont l'homme est à la fois la copule et l'instrument, le maître et l'esclave. En anthropologisant la puissance originaire de la représentation, Husserl a manqué la dimension négative de cette puissance, repérable jusque dans sa constitution transcendantale, sa face saturnienne, aveugle et fatale qu'implique nécessairement sa mise en dispositif, son Gestell, par la principialité de la raison.

La tâche de Heidegger consiste précisément à surmonter la dimension négative et saturnienne du pouvoir fondamental qui commande nos facultés de compréhension et d'action. Mais règne sur la nature de cette tâche un très grand malentendu.

La vulgate heideggérienne comprend ce surmontement comme destruction de la métaphysique, c'est-à-dire comme prise de congé radicale de tout pouvoir fondamental, ce que Reiner Schurmann appelle « le principe d'anarchie " ${ }^{17}$; cette prise de congé se manifesterait par la notion eckartienne, reprise par Heidegger, de "délaissement » (Gelassenheit) qui affranchirait l'homme de toute domination et redonnerait au monde son innocence, en défaisant les articulations fondamentales par lesquelles le système se fait dispositif de dispensation du pouvoir.

17. Reiner Schurmann, Le Principe d'anarchie, trad. franç., Paris, Seuil, 1982. 
Mais, en réalité, ce que critique Heidegger dans le principe de raison, dans le système de la subjectivité, dans le Gestell technique qu'il met en place et dans la volonté de puissance qui les commande, ce qu'il considère comme leur négatif, ce n'est pas tant leur puissance de domination et le déchaînement de leur violence que, bien au contraire, l'épuisement de la puissance qui s'y joue, sa constriction, son blocage au sens schellingien du terme et, de façon plus décisive encore, l'absence de souveraineté de cette violence dont le règne de la technique passe aux yeux de Heidegger pour le symptôme le plus évident. Sur cette question du pouvoir, Heidegger reste finalement l'héritier de la tradition philosophique, de Platon à Campanella et de Campanella à Marx, pour qui la tyrannie et la violence ne sont pas tant un excès de pouvoir que l'effet d'un pouvoir séparé de son origine et de sa puissance fondamentales.

Ainsi, il ne s'agit pas pour Heidegger de penser l'impuissance ou la nonpuissance auquel nous conduirait notre retrait par rapport au système, mais bien au contraire la surpuissance (Übermacht) qu'atteint le système lorsqu'il se trouve affranchi du principe de raison et de ce qui, dans ce principe, bloque le système, épuise le pouvoir et en perd la souveraineté. De la sorte, Heidegger n'opère pas tant la destruction de la métaphysique, qu'une destruction dans la métaphysique, c'est-à-dire la destruction de tout ce qui, dans le système, restreint et bloque la dispensation du pouvoir. À cette fin, Heidegger maintient dans sa philosophie le système et sa structure triadique originaire considérée comme l'architectonique fondamentale de toute dispensation du pouvoir; mais, pour fonder plus souverainement l'architectonique de son système, il substitue à la principialité du principe de raison, la principialité de l'Être considéré comme acte et comme puissance, qui constituent, pour Heidegger lui-même, le couple directeur de l'ontologie et de toute son histoire ${ }^{18}$. Telle est la signification profonde de sa philosophie de l'Être.

Nous montrerons d'abord pourquoi, pour Heidegger, le principe de raison et sa volonté de puissance entraînent le blocage et l'impuissance du système, puis nous verrons ensuite en quoi l'affranchissement de la triade originaire par rapport au principe de raison conduit à la souveraineté absolue de l'Être ou, plus exactement, à l'Être comme souveraineté absolue.

\section{ÉPUISEMENT}

Heidegger est assurément un penseur subtil et rusé. Dans un premier temps, il s'est ingénié à montrer en quoi la raison, sous sa figure princi-

18. M. HeIdegger, Aristote, Métaphysique, $\Theta$ 1-3. De l'essence et de la réalité de la force, trad. franç. Bernard Stevens et Pol Vandevelde, Paris, Gallimard, 1991, p. 40 sq. 
pielle, appartenait à la question du système et au déploiement de sa puissance, dénonçant ainsi la fausse innocence et le caractère sainte-nitouche de l'objectivité rationaliste et de ses formalismes. Dans un deuxième temps, après avoir ainsi enfermé ses adversaires dans une métaphysique lourde du pouvoir qui les englobe et les comprend mais qu'ils ne peuvent eux-mêmes assumer, il renverse sa position, en montrant que cette puissance, mise en œuvre par le principe de raison, est finalement un néant : une puissance diminuée, vaine et épuisée qui étouffe le système, le rend inerte et le transforme en carcan, ce que Heidegger appelle le Gestell.

Le classicisme, qu'il soit philosophique aussi bien que politique ou artistique, redoute la prolifération de l'Être et l'exubérance de sa force. Son activité de prédilection, qui symbolise le mieux son génie, est le jardinage. Tailler, couper, émonder, ligaturer, emporter, mettre sous tuteur ou sous serre sont des opérations qu'il aime particulièrement pratiquer. La métaphysique classique a ainsi cherché à domestiquer la puissance fantastique et absolue de l'omnipossibilité en jeu dans les théologies de l'expression par un certain nombre de principes - principe de raison, principe d'identité, principe de non-contradiction - destinés à régler, à tempérer et à limiter le fonctionnement du système par sa dimension logique et intelligible, en sorte que la primordialité de la sagesse tienne sous sa gourme celle de la puissance.

Heidegger reproche à ce jardinage métaphysique son manque d'ouverture. Ce type de principes enferme le système sur son propre fonctionnement rendu répétitif, prévisible, anticipable, sans capacité d'invention, de rupture et d'événement. Bref, pris dans le carcan de l'intelligibilité, le système devient inerte, rigide et statique. La pensée de son mouvement est sacrifiée au profit de la compréhension de sa structure.

Or, pour Heidegger, un système sans mouvement n'est pas simplement un système diminué, restreint et bloqué : il ne fait plus du tout système. L'inertie de la triade et de son articulation n'entraîne pas seulement leur arrêt et leur répétition, mais de façon plus radicale encore leur effondrement sur elles-mêmes. C'est en réalité le mouvement même du système qui, pour Heidegger, distingue et associe les trois éléments fondamentaux de la triade et par conséquent crée le système. Le déploiement triadique du système n'est pas un simple dispositif élémentaire, une architectonique de substances fondamentales, mais le résultat de son opération, qui dans le cours même de son mouvement, à la fois éloigne et rapproche chacun des éléments de la triade en ce que Heidegger appelle une constellation (Konstellation), c'est-à-dire à la fois et en même temps, singularise le fondement, le monde, l'homme, et les articule. Une fois ce mouvement suspendu, le système se contracte sur lui-même, les éléments se confondent : on passe de la triade fondement/monde/homme à la dyade sujet/objet puis, dans un 
second temps, de la dyade sujet/objet à la monade du dispositif ou Gestell qu'institue le devenir-technique du système et de sa métaphysique (le terme monade est entendu ici non pas au sens leibnizien d'une copule obsidionale sans porte ni fenêtre, mais au sens d'un dispositif total uniforme réduit à un seul terme). Le système se perd et, avec lui, la dispensation métaphysique du pouvoir par la triade. C'est en quoi le principe de raison marque précisément l'épuisement du pouvoir fondamental de la métaphysique par la mort du système.

Dans une première opération herméneutique, Heidegger avait donc mis l'accent sur le principe de raison, c'est-à-dire sur la principialité de la raison; il avait montré comment, par la voie du principe de raison, la dyade noétique et logique sujet/objet renvoyait à la triade cachée de la Volonté de puissance (fondement), de la Subjectivité (copule) et de la Technique (monde). Après avoir remonté la filière de la dyade à la triade, Heidegger entreprend de la redescendre jusqu'à la monade. En réalité, la triade cachée épuise et effondre le système, de la triade à la dyade sujet/objet et de la dyade à la monade du Gestell. Le principe de raison est assurément le négatif du système à la démonstration duquel Heidegger s'ingénie au cours de cette redescente. Mais pour montrer que le principe de raison est le négatif du système, il fallait auparavant expliquer en quoi, par la remontée de la filière, ce même principe appartient au système de façon consubstantielle, puisque le négatif ne travaille que de l'intérieur.

La réduction et la liquidation du système par le principe de raison s'opèrent donc en deux étapes : par le passage d'abord de la triade à la dyade, puis ensuite de la dyade à la monade.

Dans le principe de raison, le principe se réduit finalement à n'être que la condition de possibilité de la connaissance et de la représentation. Cette condition de possibilité s'est peu à peu transformée et déterminée tout au long de l'histoire de la métaphysique, d'abord comme Bien au-delà de l'Être, puis comme Sujet transcendantal, et enfin comme Volonté de Puissance. De Platon à Kant, et de Kant à Nietzsche, se constitue pour Heidegger la généalogie fondamentale de la métaphysique comme présence et représentation. Ce statut réducteur entraîne un risque de confusion entre le principe et la copule, ou, mieux encore, entre la subjectivité du système et l'égoïté de l'homme, c'est-à-dire entre, d'une part, la prise de conscience de soi du système par la dynamique même de son mouvement en un savoir absolu, et, d'autre part, l'auto-fondation ou, plus exactement, l'autoinscription de la copule dans le système. Et peu importe que cette autoinscription soit constitutive comme chez Schelling ou seulement régulatrice, réduite, comme chez Fichte, au statut d'une illusion transcendantale nécessaire à la constitution et à la mise en mouvement du système. Dans l'un et l'autre cas, c'est comme s'il n'y avait plus d'autre différence entre 
le principe et la copule que celle que la tradition kantienne institue entre l'ego transcendantal et le moi empirique. Dans ces conditions, les subtiles articulations de la triade originaire se réduisent à une brutale opposition entre un sujet qui pose les conditions de la connaissance et un objet, c'est-à-dire un étant qui tire son être et la raison de son existence du schème ainsi posé.

La dyade du sujet et de l'objet est une illusion. Elle se pense comme une instance fondatrice et configuratrice du monde alors qu'elle n'est qu'une étape dans l'effondrement du système, pas même son point d'arrivée: double illusion donc de ce qui se croit point de départ alors qu'il n'est qu'une transition, mais aussi de ce qui se juge fondation du monde alors qu'il n'est que le symptôme de la disparition et de l'effondrement du système.

Tel est le sens du devenir-technique de l'objectivité sur lequel Heidegger a tellement insisté dans son œuvre, et par « devenir-technique de l'objectivité " est entendu l'accomplissement de l'objectivité en tant que schème d'organisation de la computation universelle et de la mobilisation totale du monde. De fait, pour Heidegger, la computation algorithmique du monde et sa mobilisation technique réduisent à son tour le sujet à l'état d'objet qu'il a lui-même posé et qui finalement l'asservit aux schèmes par lesquels pourtant il prétend maîtriser le monde. Le sujet disparât dans l'objectivité qu'il a produite. La subjectivité, par la médiation de la dyade, retourne à la substance, la triade à la monade. Il n'y a plus d'articulation, donc plus de mouvement possible. Le devenir-technique de l'objectivité entraine un blocage absolu, la mobilisation totale du monde est un surplace : c'est la fin de l'histoire.

Il y a donc deux façons de penser l'essence-mouvement du mouvement, le mouvement du mouvement, dans le système : la mobilisation totale du Gestell, d'une part, et la Bewegung pure et absolue de la constellation, d'autre part. Entre les deux s'installe une véritable gigantomachie. La Mobilmachung renvoie à l'étymologie latine de la notion de mouvement : movere - mobilis. Le terme de mobilis réfère à son tour à la summa divisio du droit romain entre immeubles et meubles, entre bien-fonds et biens instables. Derrière la Mobilmachung, se profile le monde de l'échange monétaire, du droit commutatif et de 1'agir catallactique. Or, ce mouvement est un leurre. Loin de déplacer le système, il le fait retomber et s'effondrer sur lui-même. La Mobilmachung est en quelque sorte la figure inversée du principe aristotélicien : tandis que la noésis noéseôs de nature immobile meut véritablement le monde en une immobile mobilité, la Mobilmachung du principe de raison, qui est pourtant de nature mobile, finit par l'immobiliser en une mobile immobilité. On songe à la fameuse formule du prince de Lampedusa qui exprime mieux que toute autre l'intelligence du conservatisme : « Pour que rien ne change, il faut que tout change. " À la Mobil- 
machung, Heidegger oppose la notion germanique du Weg ou chemin qu'il décline en de nombreux paronymes. Ce chemin est celui qu'emprunte à l'aventure, au risque de la perte et de l'errance, le Wanderer, le promeneur, sans but ni raison, sans pourquoi ni comment. Ce cheminement sans but ni raison produit un mouvement spécifique, la Bewegung. C'est sur ce chemin que nous allons désormais nous engager pour mieux comprendre le sens de cette dernière notion.

\section{INNOCENCE}

Les textes de Heidegger sur le principe de raison ne se contentent pas de faire la généalogie et la critique des philosophies de la représentation et de l'objectivité. À ce mode de connaissance et mieux encore d'inscription de l'homme dans le système, il propose pour alternative une autre épistémologie, fondée non pas sur la question du «pourquoi » (Warum), mais sur le constat du "parce que» (Weil). Heidegger commente les fameux vers d'Angelus Silesius :

La rose est sans pourquoi, fleurit parce qu'elle fleurit, N'a souci d'elle-même, ne désire être vue ${ }^{19}$,

par d'autres vers, ceux de Goethe qu'il cite dans sa conférence de 1956 sur le principe de raison:

Comment? Quand? Où? Les dieux restent muets.

Tiens-t'en au parce que, ne demande pas le pourquoi ${ }^{20}$.

Heidegger ne fait finalement ici que reprendre la distinction classique dans l'Analytique aristotélicienne entre la démonstration de causalité, démonstration dite dioti ou propter quid, et la démonstration d'existence dite démonstration quia: distinction qui dans l'épistémologie scolastique sépare clairement la science de l'histoire naturelle. Cette distinction logique et épistémologique entraîne une distinction métaphysique de plus grande conséquence : la distinction ou plus encore la dissociation entre le fond et le principe.

Le fond n'est pas fondement, mais assiette. Le fond, c'est le fond des choses, ce par quoi les choses tiennent et se maintiennent, ne relevant d'autre principe que d'elles-mêmes. Le fond assure la pose et le repos de la chose, sans entraîner la moindre dépendance à un fondement qui l'arrai-

19. Le Principe de raison, p. 103.

20. Le Principe de raison, La conférence, p. 264-265. 
sonnerait. La connaissance a alors pour but de laisser éclore la chose en ce qui lui est propre, sans la référer à quelque instance générale transcendante ou immanente, et ainsi de l'asseoir en son assiette. Â ce fond singulier des choses répond un principe général : l'Être. Mais chez Heidegger l'Être ne fonde pas; il assure non pas l'assise des choses mais leur mouvement; or c'est parce que le principe est mouvement et non pas fondement qu'on ne peut référer la chose à ce principe ni en rendre raison au nom de ce principe.

La connaissance de la chose par son «parce que » prétend « laisserêtre » la chose. Telle est la vocation de la parole ou du logos grec que Heidegger oppose à la ratio latine de la raison-calcul.

«Le grec legein ne signifie pas seulement parler et dire, ne signifie même pas simplement recueillir, mais, nous faisant signe d'encore plus loin, ne recueille que pour remettre et pour laisser à lui-même ce qui est recueilli en respectant en lui le repos ou la pose qui lui est le plus propre ${ }^{21}$."

Par cette approche délaissante de la chose, la connaissance semble atteindre à l'innocence du savoir.

L'innocence du savoir aiguillonne, sous diverses exigences, toutes les formes d'épistémologie, phénoménologiques aussi bien qu'objectivistes et formalistes. Mais que faut-il entendre par « innocence du savoir »? Innocence provient du latin In-nocere. In-nocere signifie : « ne pas nuire », ne pas soumettre le monde à des effets de pouvoir ni l'inscrire dans des rapports de force; ce qui signifie pour l'homme à la fois ne pas lui imposer ses perspectives, mais aussi ne pas chercher à y puiser sa force. Un savoir innocent est donc un savoir qui ne cherche ni à arraisonner les choses en vue de quelque intérêt ou stratégie humaine, ni à inscrire l'homme par la médiation du savoir dans un dispositif de dispensation de pouvoir. De prime abord, il peut sembler que l'épistémologie heideggérienne du « parce que ", du recueillement, du laisser-être et du délaissement retire les choses de tout horizon de puissance et par là même prend congé du système dispensateur de pouvoir. $S$ 'il en était ainsi, les troisième et quatrième thèses de cet article sur le rapport que Heidegger entretient à la question du système perdraient toute validité. Mais il faut se défier de l'apparence bonnasse et débonnaire de l'approche heideggérienne qui dissimule en réalité un rapport intensifié à la puissance. Loin de retirer l'homme hors du système, l'épistémologie du délaissement n'est en réalité qu'une stratégie pour son réarmement.

21. Jean Beaufret, in Le Principe de raison, p. 27. 


\section{SOUVERAINETÉ}

Heidegger conclut Le Principe de raison par la fameuse parole d'Héraclite, mystérieuse et poétique, qui résume parfaitement le projet heideggé-

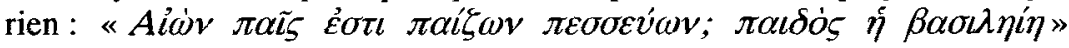
(L'Aiôn est un enfant qui joue au tric-trac. C'est à un enfant qu'appartient la souveraineté) $)^{22}$. "Sous la figure de l'enfant-roi héraclitéen, Heidegger manifeste la coappartenance essentielle de l'innocence et de la puissance.

L'Aiôn, ce que les Latins appellent aevum, constitue dans la pensée des Anciens une notion fondamentale qui dépasse sa simple signification temporelle ou supratemporelle; elle a rapport en réalité à l'énergie cosmique, à son rythme et à sa dispensation. Heidegger incorpore l'Aiôn dans son ontologie et le traduit ici comme « dispensation de l'Être» (Seinsgeschick). En tant qu'énergie, rythme et dispensation, l'Aiôn désigne en réalité l'articulation même de la triade. La dispensation de l'Être ou mieux encore sa condition de possibilité qu'est l'articulation de la triade doit donc être, pour Heidegger, comparée à un enfant qui joue.

La dispensation de l'Être, l'articulation entre le principe, le monde et l'homme, le fonctionnement du système sont un jeu. Et s'il faut parler de tric-trac ${ }^{23}$ et non pas de dames, c'est que le tric-trac avec son cadran, ses dés, son circuit, son jeu de blocage et de mouvement, de position et de substitution, est une métaphore du système bien plus évidente que tout autre jeu. Or, le jeu exprime précisément le fonctionnement du système libéré du principe de raison.

"L'enfant joue le jeu du monde. Il joue parce qu'il joue. Le " parce que" disparaît dans le jeu. Le jeu est sans pourquoi [...] Le jeu seul demeure : il est ce qu'il y a de plus haut et de plus profond ${ }^{24}$. "

Et Heidegger précise dans «Identité et différence »: «L'essence de l'Être est le jeu lui-même ${ }^{25}$. »

22. Héracute, Fragment 52, cit. in Le Principe de raison, p. 243.

23. On renvoie habituellement dans la sentence d'Héraclite le participe présent « $\pi \varepsilon \sigma \sigma \varepsilon v ́ \omega v$ au jeu de dames, parfois de façon plus vague et générique aux dés. Heidegger traduit le terme héraclitéen par une expression elle aussi générale Brettspiel qui désigne tous les jeux à damier ou à plateau. En réalité, $\delta \pi \varepsilon \sigma \sigma o ́ s$ possède en grec une signification bien précise : il désigne les petites pierres ovales en forme de gland que les Grecs employaient en guise de jetons au jeu de tric-trac. Le tric-trac - ce qui était appelé quand j'étais enfant le jacquet, et qui aujourd'hui est plus connu sous son nom anglais de backgammon - est un jeu répandu depuis toujours dans tout le bassin méditerranéen.

24. Le Principe de raison, p. 243.

25. M. HeidegGer, "Identité et différence ", in Questions I, trad. franç. A. Préau, Paris, Gallimard, 1968, p. 300. 
Le jeu marque l'affranchissement de tout déterminisme de la relation, voire de toute relation déterminée entre le principe, le monde et l'homme. Cet affranchissement entraîne à son tour un éloignement, une mise à distance, une différence radicale entre les éléments de la triade. L'éloignement qu'entraîne la suspension du principe de raison et du principe d'identité ne remet pourtant pas en cause la coappartenance systématique des éléments. Certes, l'égalité et la convertibilité automatique des éléments de la triade entre eux ne vont plus de soi, mais ils continuent pourtant à appartenir au même dispositif, à la mêmeté et à l'identité de l'Être, de la pensée et du monde. La différence, selon Heidegger, renvoie à une identité plus originaire que le principe d'identité, une identité antérieure à l'Être lui-même et qui le constitue, ce que Heidegger appelle mêmeté (Selbstheit) ou coappartenance (Zusammengehörigkeit) pour les distinguer et les opposer à l'égalité (Gleichheit) et à la commutativité du principe d'identité tel qu'il se présente sous sa formule canonique $A=A^{26}$. En effet, l'éloignement de la différence permet de restituer la triade à elle-même par rapport à la confusion de la monade. Mieux encore, il dramatise et intensifie la possibilité de leur rapprochement. C'est du plus lointain et du plus étranger que provient ce qui nous est le plus proche. Simplement, l'articulation du plus lointain, de ce qui semble incommensurable, ne se commande plus au nom de quelque nécessité déterminée par la raison et la logique. Elle dépend bien plutôt d'une libre faveur, du libre jeu (d'où le coup de dés qu'on donne au tric-trac) des trois éléments entre eux. Le libre jeu et la libre faveur, qui rapprochent et articulent du plus lointain, à la limite de l'incommensurable et de la dissociation, les éléments fondamentaux de la triade, Heidegger les appelle Ereignis : à la fois événement, surgissement et appropriation. Ce qui est ainsi toujours à la limite du démantèlement du système est aussi l'occasion de son déploiement, de son amplification et de son fonctionnement maximum.

Mais en quoi ce jeu est-il souverain? En quoi l'enfant qui pousse ses pions sur le cadran du tric-trac est-il un enfant-roi? En quoi la libre faveur qui règne entre les éléments de la triade entretient-elle un rapport privilégié avec la question de la puissance de l'Être et de sa dispensation? En quoi l'essence même de l'Être, s'il est vrai comme l'affirme Heidegger que cette essence est le jeu, se trouve-t-elle déterminée par la souveraineté que le jeu déploie dans le système? En quoi, enfin, l'Ereignis qui résulte du jeu instaure-t-il le règne souverain de l'Être et de son Übermacht?

26. Le Principe de raison, p. 262 : «L'Être est défini à partir d'une identité et comme un trait de cette identité. Plus tard, au contraire, la métaphysique a représenté l'identité comme un trait de l'Être [...] L'identité de la Pensée et de l'Être qui parle dans la sentence de Parménide

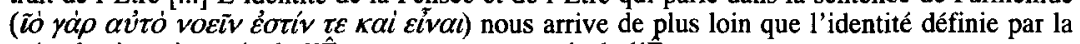
métaphysique à partir de l'Être et comme un trait de l'Etre. » 
En tant qu'événement, surgissement et appropriation, l'Ereignis est une figure de l'acte; mais cette figure se distingue singulièrement des autres conceptions de l'acte que propose la métaphysique dans son histoire. L'Ereignis est étrangère à l'entéléchie aristotélicienne, qui reste corsetée et soumise aux limites de sa puissance qu'elle réalise sans jamais pouvoir la déborder. De fait, l'Ereignis de son côté n'est absolument pas limité par quelque champ de possibilité. L'Ereignis surprend ce qui est de l'ordre du possible et du probable en provenant de son dehors, d'un lieu non repéré dans le champ de la possibilité, c'est-à-dire de l'impossible. C'est en quoi l'Ereignis est précisément événement et surgissement. Ainsi définie, la notion heideggérienne d'Ereignis semble devoir beaucoup à l'actualisme de Gentile et à sa pensée de l'acte pur $^{27}$. Pourtant ce qui distingue radicalement l'Ereignis de l'acte pur, c'est que l'Ereignis ne prétend pas fonder, c'est-à-dire ouvrir et capter un nouveau champ de possibles à partir de sa provenance impossible, de l'impossibilité de sa provenance. Acte et puissance s'affranchissent ainsi de leurs entredéterminations contraignantes. Sous la figure de l'Ereignis, l'acte se libère de tout conditionnement par la possibilité, mais il libère aussi la puissance de son obligation à être, à se réaliser et à s'épuiser dans et par l'effectivité d'un acte. Sans pour autant que cette entrelibération de l'acte et de la puissance remette en cause, et c'est le tour de force de Heidegger, leur coappartenance. Au contraire, de cette façon le cercle aristotélicien de l'acte et de la puissance reste maintenu, mais sous une forme dynamique et non plus statique. C'est en tant que tel que l'Être devient le mouvement du jeu souverain (Bewegung dieses hohen Spieles) de la distribution et du retrait.

Libérée de son devoir-acte, la puissance en effet non pas se réalise mais au contraire se retire dans le mouvement même de surgissement de l'Ereignis. Ce qui se retire se met, selon l'expression de Heidegger, en réserve. La puissance n'est rien d'autre que la réserve de ce qui s'est retiré au moment du surgissement de l'acte. Or, la réserve permet à l'Ereignis de relancer à tout moment son mouvement hors de toute fondation, sans pour autant que cette réserve cherche à le déterminer, préservant bien plutôt sa liberté et sa gratuité. À son tour l'Ereignis déploie la réserve sans pour autant la manifester ni l'épuiser. Ainsi, plus il y a de réserve, plus il y a de libre et d'imprévisible surgissement, et plus il y a de surgissement libre et imprévisible, plus il y a de mise en réserve. Alors se mettent en place une véritable échelle de perroquet, un cercle dynamique où la puissance et l'acte s'intensifient mutuellement à l'infini. Ce nouveau cercle, cercle d'entrelibération et non d'entredétermination entre l'acte et la puissance,

27. Sur ces questions, voir Charles AlunNI, « Giovanni Gentile-Martin Heidegger. Note sur un point de (non) traduction, Cahiers du Collège international de philosophie, 6, 1988, p. 7-12, et ID., "Giovanni Gentile ou l'interminable traduction d'une politique de la pensée ", Lignes, 4, 1988, p. 181-194. 
entre le surgissement et la réserve, engendre la souveraineté du système. Sont souverains ici non seulement l'inviolabilité et l'imprescriptibilité, au sens aussi bien juridique que métaphysique des termes, que la réserve assure à la dispensation, mais aussi la décision, au sens schmittien du terme, de l'Ereignis dans son surgissement. Par cette synthèse de l'inviolabilité et de la décision, de la réserve et du surgissement, de la toutepuissance et de l'acte pur, nous atteignons à la souveraineté absolue du système et de son mouvement.

Ainsi, l'épistémologie du laisser-être que Heidegger oppose au principe de raison et à la mobilisation totale du monde par la technique est une fausse ingénue. Elle présente une innocence de façade. En réalité, sous cette notion de laisser-être, Heidegger thématise l'expérience du savoir nécessaire à l'homme pour participer à la souveraineté ludique du système et y jouer son rôle de copule, de même que le principe de raison entraîne l'homme-sujet dans la mobilisation totale du monde par la technique. L'homme est alors jeté et entraîné dans le pur mouvement du Jeu souverain. Or, ce mouvement perd vite son caractère de cheminement bucolique sur les chemins forestiers de traverse. Sur son passage tout est dévasté. Le pur mouvement de la Bewegung n'est rien, mais transit toutes choses jusque dans son retrait, sans demeurer en aucune. Il est parent de ces dieux guerriers et vengeurs qui, avant de se réfugier dans les nuées de l'empyrée, se présentaient, selon la puissante métaphore de Naphta dans la Montagne magique, comme des boules de feu derrière lesquelles s'avançaient leurs armées. Lorsque l'enfant joue, non seulement il supprime le principe de raison et son pourquoi, mais plus encore - et l'aveu de Heidegger est ici patent -, il fait disparaître le " parce que », c'est-à-dire le quia des choses, leur assiette et leur constance, de sorte que «le jeu seul demeure ». Le pur mouvement, en traversant les étants, les spectralise. Le monde devient fantomatique. Ce n'est qu'à ce prix que le mouvement atteint à la souveraineté absolue.

Pour penser l'authentique innocence du savoir, c'est-à-dire un savoir affranchi de tout rapport à la question de la puissance, il faut se retourner vers Nietzsche à qui Heidegger emprunte les notions de jeu et d'innocence. En effet, par le jeu et l'innocence, Nietzsche a de son côté entrepris de s'affranchir véritablement de toute ontothéologie, c'est-à-dire de toute pensée de la dispensation, par la médiation du système et de sa triade, de l'Être comme pouvoir, du pouvoir comme Être. Le sens du différend (Auseinandersetzung) que Heidegger entretient avec Nietzsche sur la question de la métaphysique, de sa puissance et de son achèvement s'en trouve alors radicalement bouleversé. Mais cette question appelle une autre explication.

Pierre CAYE (avril 1998). 